\title{
Identification and Mapping of Adult Plant Resistance Loci to Leaf Rust and Stripe Rust in Common Wheat Cultivar Kundan
}

Y. Ren, Mianyang Institute of Agricultural Science/Mianyang Branch of National Wheat Improvement Center, Mianyang 621023, Sichuan, China; R. P. Singh, B. R. Basnet, and C. X. Lan, International Maize and Wheat Improvement Center (CIMMYT), 06600 México D.F., Mexico; J. Huerta-Espino, Campo Experimental Valle de México INIFAP, 56230 Chapingo, Edo. de México, Mexico; E. S. Lagudah, CSIRO Agriculture, Canberra, ACT 2601, Australia; and L. J. Ponce-Molina, National Institute of Agricultural and Livestock Researches (INIAP-Ecuador), Santa Catalina Experimental Station, Quito, Ecuador

\begin{abstract}
Leaf rust (LR) and stripe rust (YR) are important diseases of wheat worldwide. We used 148 recombinant inbred lines (RIL) from the cross of Avocet $\times$ Kundan for determining and mapping the genetic basis of adult plant resistance (APR) loci. The population was phenotyped LR and YR for three seasons in field trials conducted in Mexico and genotyped with the diversity arrays technology sequencing (DArT-Seq) and simple sequence repeat markers. The final genetic map was constructed using 2,937 polymorphic markers with an average distance of 1.29 centimorgans between markers. Inclusive composite interval mapping identified two co-located APR quantitative trait loci (QTL) for LR and YR, two LR QTL, and three

YR QTL. The co-located resistance QTL on chromosome 1BL corresponded to the pleiotropic APR gene Lr46/Yr29. QLr.cim-2BL, QYr.cim$2 A L$, and $Q Y$ r.cim-5AS could be identified as new resistance loci in this population. $L r 46 / Y r 29$ contributed 49.5 to 65.1 and 49.2 to $66.1 \%$ of LR and YR variations, respectively. The additive interaction between detected QTL showed that LR severities for RIL combining four QTL ranged between 5.3 and $25.8 \%$, whereas the lowest YR severities were for RIL carrying QTL on chromosomes $1 \mathrm{BL}+2 \mathrm{AL}+6 \mathrm{AL}$. The high-density DArT-Seq markers across chromosomes can be used in fine mapping of the targeted loci and development SNP markers.
\end{abstract}

Leaf (brown) rust (LR) and stripe (yellow) rust (YR), caused by Puccinia triticina and $P$. striiformis f. sp. tritici, respectively, are fungal diseases of common wheat (Triticum aestivum L.) that significantly reduce wheat production worldwide. Epidemics of wheat rusts can occur on a continental scale due to the widespread dispersal of urediniospores, which rapidly destroy leaf tissue and significantly reduce grain yield and quality (Khan et al. 2013; Roelfs et al. 1992; Wellings 2011). LR causes wheat yield losses ranging from 10 to 50\% (Huerta-Espino et al. 2011), whereas losses from YR can range from 18 to $90 \%$ (Chen 2014), depending on the susceptibility of the cultivar, timing of the initial infection, rate of disease development, and duration of disease. Although wheat rusts can be managed through fungicide use, its effectiveness is limited by financial constraints, chemical availability, and inclement weather conditions affecting timely application. Therefore, growing resistant cultivars is the most economical and environment-friendly approach to managing these diseases.

Race-specific and race-nonspecific resistances are the two main types of resistance to wheat rusts. Race-specific resistance is generally qualitatively inherited and often confers protection throughout the growth stages. Therefore, this type of resistance is also called all-stage resistance (Chen 2013). It is usually short-lived due to the high evolutionary potential of the pathogen (Huerta-Espino et al. 2011; Wellings 2011). In contrast, adequate levels of racenonspecific resistance are usually quantitatively inherited and involve multiple genes, contributing minor to intermediate effects. Wheat lines carrying these resistance genes are usually susceptible at the seedling stage but express a slow-rusting type of resistance at the postseedling growth stages of wheat plants. This type of resistance is also often called adult plant resistance (APR) (Chen 2005;

Corresponding author: C. X. Lan; E-mail: c.lan@ cgiar.org

*The $\boldsymbol{e}$-Xtra logo stands for "electronic extra" and indicates that one supplementary figure and one supplementary table are published online.

Accepted for publication 16 November 2016.

This article is in the public domain and not copyrightable. It may be freely reprinted with customary crediting of the source. The American Phytopathological Society, 2017.
Lagudah 2011; Lin and Chen 2007; Roelfs and Bushnell 1985). Numerous resistance genes to LR and YR have been identified and cataloged in wheat (McIntosh et al. 2014). Among these, the three pleiotropic APR genes reported in wheat- Lr34/Yr18/Pm38/Sr57/Ltn1, Lr46/Yr29/Pm39/Sr58/Ltn2, and Lr67/Yr46/Pm46/Sr55/Ltn3-confer resistance to LR, YR, powdery mildew, and stem rust, and are also associated with the morphological trait postflowering leaf tip necrosis (LTN) (Helguera et al. 2003; Herrera-Foessel et al. 2010, 2014; Lillemo et al. 2013; Singh et al. 2011; William et al. 2003). The effect of these pleiotropic APR genes is moderate when present alone but they play an important role in gene combinations and interactions with additive effects, resulting in high levels of durable resistance. In general, accumulating four to five APR genes into a single genotype can reach "near-immunity" or a high level of resistance (Singh et al. 2000).

Closely linked molecular markers can be used to pyramid corresponding APR genes into elite wheat lines to achieve a high level of resistance (Chen 2013; Collard and Mackill 2008; Yang et al. 2012). In the last 30 years, several polymerase chain reactionbased marker systems such as amplified fragment length polymorphism (AFLP), random amplified polymorphic DNA, resistance gene analog polymorphism, simple sequence repeat (SSR), and sequence-tagged site molecular marker systems have been implemented in wheat rust genetics and breeding research ( $\mathrm{Li}$ et al. 2014; Rosewarne et al. 2013). However, next-generation sequencing (NGS) technologies provide better opportunities to unravel the genetic bases of quantitative traits by providing large amounts of data points in a cost- and time-effective manner (Elshire et al. 2011; Yang et al. 2012). Diversity arrays technology sequencing (DArT-Seq), a new approach based on traditional DArT-array and NGS techniques, was developed as a simple, rapid, and robust approach for reducedrepresentation sequencing of multiplexed samples that combines genome-wide molecular marker discovery and genotyping (http:// www.diversityarrays.com/dart-application-dartseq). The advantages of DArT-Seq over the DArT-array version are the sequence of the genomic representations and the development of high-density linkage maps. Therefore, this technology accelerates high-resolution mapping and detailed genetic dissection of traits (Raman et al. 2014).

'Kundan' wheat (syn. 'DL153-2') has remained resistant to LR and YR for more than three decades (Dhanda and Sethi 2002). It is a semidwarf wheat cultivar released in 1985 in India under rainfed conditions by the Indian Agricultural Research Institute (Grewal et al. 2007). 
Investigating the genetic basis of resistance in Kundan could be of importance to developing new resistant advanced wheat lines or varieties with higher yields and adaptation.

The objectives of our study were to (i) investigate the genetic basis of LR and YR resistance in an 'Avocet' $\times$ Kundan recombinant inbred line (RIL) population, (ii) identify co-located APR loci for LR and YR using molecular markers, and (iii) determine the additive effects between detected LR and YR quantitative trait loci (QTL).

\section{Materials and Methods}

Plant materials. In total, $148 \mathrm{~F}_{5}$-derived $\mathrm{F}_{6} \mathrm{RIL}$ were developed from a cross between Kundan and the $Y r A$-lacking selection Avocet- $Y r A$ (hereafter referred to as Avocet). Kundan (pedigree: Tanori 71/NP 890) shows susceptibility in seedlings and high levels of APR to current races of Mexican YR and LR fungi, whereas Avocet is susceptible to these races at all growth stages. The RIL population was developed using a single-head descent approach, as described by Basnet et al. (2013). After multiplication of the $\mathrm{F}_{6}$ RIL and parents at El Batan, Mexico, the same source of seed was used for genotyping and all of the phenotyping experiments.

Field evaluations for characterizing $L R$ and YR resistance. The RIL and parents were evaluated for APR to LR in Yaqui Valley near Ciudad Obregon, Mexico, in the 2012-13 and 2013-14 growing seasons (hereafter referred as LR2013Y and LR2014Y experiments, respectively) and at El Batan in 2014 (LR2014B). Similarly, three YR experiments were conducted at the Toluca, Mexico research station during the 2012, 2013, and 2014 crop seasons (YR2012, YR2013, and YR2014 experiments, respectively). Field plots consisted of $0.7-\mathrm{m}$ paired rows with approximately 60 plants of each line. Avocet near-isolines (NIL) carrying $Y r 24$ and $Y r 26$ were used as spreaders in LR studies whereas a mixture of 'Morocco', Avocet NIL carrying $Y r 31$, and six lines possessing the $Y r 27$ gene derived from the cross Avocet $\times$ 'Attila' were used as YR spreaders. Spreaders were planted around the experimental area and as hill plots in the middle of a $0.3-\mathrm{m}$ pathway on one side of each experimental plot. An LR epidemic was initiated by spraying an equal mixture of $P$. triticina races MBJ/SP and MCJ/SP onto LR spreader, suspended in Soltrol 170. The equal mixture was prepared by suspending the same amount of each race inoculum (around 3 $\mathrm{g} / \mathrm{race}$ ) in 1 liter of Soltrol 170 oil. The avirulence and virulence formulas of MBJ/SP and MCJ/SP were described by Herrera-Foessel et al. (2012). Spreaders for YR studies were sprayed in a similar manner with a mixture of $P$. striiformis races Mex96.11 and Mex08.13. Although Mex96.11 is virulent to $\operatorname{Yr} 27$ and avirulent to $\operatorname{Yr} 31$ and Mex08.13 is avirulent to $Y r 27$ and virulent to $Y r 31$, both parents were susceptible to these isolates at the seedling stage in greenhouse experiments. Four races are predominantly prevalent in Mexico.

Disease severities (DS) on parents and RIL were recorded at least twice according to the modified Cobb's Scale (Peterson et al. 1948) and the host reaction was scored as described by Roelfs et al. (1992). In instances of repeated DS data, the first note was recorded when the susceptible parent Avocet displayed at least $70 \%$ severity at milk stage onward for Avocet and middle dough stage for Kundan, and was continued at weekly intervals until it reached 90 to $100 \%$. For multiple disease readings, the area under the disease progress curve (AUDPC) value was calculated following Bjarko and Line (1988).

Genetic and statistical analysis. The RIL and their parents were evaluated with an augmented complete randomized design in all experiments (Federer 1956, 1961), where two parents and 28 RIL were replicated twice and randomly distributed across the experimental field. Based on DS and infection responses, RIL were classified into three different phenotypic categories according to Singh and Rajaram (1992): homozygous parental type resistant (HPTR), homozygous parental type susceptible (HPTS), and lines with different responses from the two parents (OTHER).

The number of LR and YR APR genes in the Avocet $\times$ Kundan population was estimated using Mendelian segregation analysis (Knott and Padidam 1988; Singh and Rajaram 1992), where the observed frequencies for each category (HPTR:HPTS:OTHER) were tested against the expected frequencies for different numbers of additive genes using $\chi^{2}$ analysis. Moreover, the minimum number of APR genes was also estimated using the quantitative approach described by Wright (1968) as $n=$ $(\mathrm{GR})^{2} / 4.27\left(\sigma^{2} \mathrm{~g}\right.$ ), where GR (genotypic range) $=$ phenotype range $\times$ narrow-sense heritability $\left(h^{2}\right), h^{2}=\sigma^{2} \mathrm{~g} /\left(\sigma^{2} \mathrm{~g}+\sigma^{2} \mathrm{e}\right), \sigma^{2} \mathrm{~g}=$ genetic variance of the $\mathrm{F}_{6} \mathrm{RIL}$ in the present population, and $\sigma^{2} \mathrm{e}=$ error variance. Analysis of variance was carried out using SAS 9.2 (SAS Institute, Cary, NC) with a program from Scott and Milliken (1993) based on the final disease severity (FDS) in each environment.

Molecular markers, linkage map construction, and QTL analysis. The DNA of parents and RIL were extracted using the cetyltrimethylammonium bromide method described in the molecular breeding manual by the International Maize and Wheat Improvement Center (CIMMYT 2005). Two parents and the 131 RIL, selected for homozygosity of phenotypic responses, were analyzed with 50,000 DArT-Seq platforms by DArT Company in Australia and 176 polymorphic SSR markers in the International Maize and Wheat Improvement Center (CIMMYT) Biotech laboratory. Finally, 2,937 polymorphic markers without redundant alleles were used to construct the linkage maps with Joinmap 4.1 (Van Ooijen 2006). Linkage maps were graphically visualized with MapChart (Voorrips 2002). The order of markers and the chromosomal assignments of the linkage groups were based on a wheat consensus genetic map and physical bin location of DArT markers (Francki et al. 2009; Huang et al. 2012; Somers et al. 2004; Wilkinson et al. 2012). QTL mapping for FDS and AUDPC (namely, AYR2013Y is the AUDPC in Obregon in the 2013 crop season) from each experiment and mean of FDS for both LR and YR diseases (LRM and YRM, respectively) across experiments was carried out using inclusive composite interval mapping (ICIM) (Meng et al. 2015). The QTL results have also been confirmed by QTL Cartographer software (Wang et al. 2012). The logarithm of odds (LOD) threshold to declare significant QTL at the $P=0.05$ for each trait was determined based on 1,000 permutation tests. Stepwise regression was used for detecting the percentages of phenotypic variance explained by individual QTL and additive effects at the LOD peaks. QTL designations were assigned following the recommended practices (http://wheat.pw.usda.gov/ggpages/wgc/ 98/Intro.htm).

Table 1. Adult plant disease responses in Mexico of parents and Avocet $\times$ Kundan recombinant inbred lines (RIL) when evaluated to leaf rust (LR) for two seasons in Ciudad Obregon (LR2013Y and LR2014Y) and one season in El Batan (LR2014B), and to stripe rust (YR) during three seasons (YR2012, YR2013, and YR2014) in Toluca, with estimated minimum number of resistance genes using the Wright method

\begin{tabular}{|c|c|c|c|c|c|c|}
\hline Parent, parameter & LR2013Y & LR2014Y & LR2014B & YR2012 & YR2013 & YR2014 \\
\hline Avocet $^{\mathrm{a}}$ & $90 \mathrm{~S}$ & $100 \mathrm{~S}$ & $90 \mathrm{~S}$ & $90 \mathrm{MSS}$ & $90 \mathrm{~S}$ & $90 \mathrm{~S}$ \\
\hline Kundan $^{\mathrm{a}}$ & $5 \mathrm{MSS}$ & $10 \mathrm{MSS}$ & $5 \mathrm{MSS}$ & 5 RMR & 10 RMR & 10 RMR \\
\hline RIL mean & 35.6 & 50.0 & 34.9 & 40.5 & 51.2 & 51.4 \\
\hline Range low & 1.0 & 1.0 & 1.0 & 1.0 & 5.0 & 5.0 \\
\hline Range high & 90.0 & 90.0 & 80.0 & 100.0 & 100.0 & 100.0 \\
\hline$\sigma^{2} \mathrm{~g}$ & 407.3 & 429.9 & 307.6 & 566.6 & 597.6 & 479.8 \\
\hline$\sigma^{2} \mathrm{e}$ & 31.8 & 75.0 & 54.2 & 56.4 & 33.8 & 65.5 \\
\hline$h^{2}$ & 0.93 & 0.85 & 0.85 & 0.91 & 0.95 & 0.88 \\
\hline Genes $(n)$ & 3.7 & 3.1 & 3.4 & 3.4 & 3.2 & 3.4 \\
\hline
\end{tabular}

a Parental severities are accompanied with corresponding infection responses: RMR = resistant to moderately resistant, MSS = moderately susceptible to susceptible, and $\mathrm{S}=$ susceptible. 


\section{Results}

Phenotypic evaluation in the adult plant stage. The FDS (\%) and reaction to LR were 90 to $100 \%$ with response of susceptible for Avocet and 5 to $10 \%$ with response of moderately susceptible to susceptible for Kundan during the three crop seasons. LRM of RIL ranged from 34.9 to $50.0 \%$ in the three experiments (Table 1). The frequency distribution of RIL for LR severities showed continuous and approximately normal distributions over 3 years (Supplementary Fig. S1A), indicating the quantitative inheritance of APR to LR. Approximately three to four LR APR genes were estimated based on the Wright's method (Table 1) and Mendelian segregation analysis (Table 2).

The FDS (\%) and infection responses to YR for Avocet and Kundan were $90 \%$ with response of susceptible and 5 to $10 \%$ with response of resistant to moderately resistant, respectively, during the three crop seasons. YRM of RIL ranged from 40.5 to $51.4 \%$ across 3 years (Table 1). Quantitative inheritance of APR to YR was also observed according to the continuous and approximately normal distributions of RIL for YR severities over three environments (Supplementary Fig. S1B). In addition, genetic analysis showed that four YR genes contributed to APR in the Avocet $\times$ Kundan population based on both qualitative and quantitative estimates (Tables 1 and 2).

The Pearson correlation coefficients $(r)$ of DS between 148 RIL were high among 3 years for LR $(r=0.79$ to $0.83, P<0.0001)$ and YR $(r=0.87$ to $0.90, P<0.0001$ ) (Table 3 ). These results indicated that the experimental conditions and testing environments were well controlled and consistent with an insignificant experiment-genotype interaction. In addition, significant correlations were also found between LR and YR severities ( $r=0.63$ to $0.77, P<0.0001$; Table 3 ), indicating the presence of potential co-located APR genes to both rusts in Avocet $\times$ Kundan population.

Linkage map construction. Approximately 50,000 DArT-Seq markers covering all 21 chromosomes were used in genotyping the selected 131 RIL and parents. In total, 9,474 polymorphic markers (18.9\%) were explicitly observed between the parents. Of these polymorphic markers, 6,595 (69.6\%) redundant markers were excluded by the BIN tool of IciMapping 4.1. Finally, 2,879 (5.8\% of total) DArT-Seq markers and 58 SSR markers were used in developing the whole genomic linkage maps. Forty-one linkage groups were created with 2,589 mapped markers, representing each wheat chromosome (Supplementary Table S1). The genetic linkage map covered 1,500.5, 1,519.0, and 308.8 centimorgans (cM) of genomes, A, B, and $\mathrm{D}$, respectively, with an average marker distance of $1.29 \mathrm{cM} /$ marker. Only the genetic linkage maps related to the location of detected QTL are reported herein.

QTL mapping for co-located resistance loci. Two co-located resistance loci for LR and YR were detected in Avocet $\times$ Kundan RIL using ICIM with 1,000 permutations. The most important QTL was located at the same chromosome position as the known pleotropic APR gene $L r 46 / Y r 29$ according to the tightly linked marker csLV46G22. It was detected in the resistant parent Kundan and explained 49.5 to 65.1 and 49.2 to $66.1 \%$ of LR and YR severities variations, respectively (Table 4). This locus was located in the interval of markers 1092272 and 102414 within $1.2 \mathrm{cM}$ on chromosome 1BL (Fig. 1A) and had a major effect on both rusts across all field trials with high LOD scores (22.4 to 32.4 for LR and 24.8 to 36.5 for YR). The second co-located resistance QTL derived from susceptible parent Avocet, QLr.cim-6ALQYr.cim-6AL, was closely linked to marker 1021176_60: $T>G$ on chromosome $6 \mathrm{AL}$ (Fig. 1B). It explained 8.5 to $10.9 \%$ of LR variations in LR2013Y and LR2014Y and 9.0 to 12.6\% of YR variations in YR2013 and YR2014, respectively (Table 4).

QTL mapping for APR to LR. Two APR LR QTL, identified on chromosomes 2BL and 2DS based on ICIM analysis, were designated as QLr.cim-2BL (Fig. 1C) and QLr.cim-2DS (Fig. 1D), respectively. QLr. cim-2BL was located between 1237388 and 1081780_35:C $>T$. This QTL was detected in the LR2013Y and LR2014Y and it explained 4.1 to $5.6 \%$ of the LR variation (Table 4). It was derived from Avocet. Similarly, QLr.cim-2DS was flanked by DArT-Seq markers 1020115 and 1242814 and explained 6.4 to $7.8 \%$ of LR variation in the LR2013Y (Table 4). The resistance allele of this QTL was contributed by Kundan.

QTL mapping for APR to YR. Three YR resistance QTL were detected in the Avocet $\times$ Kundan population. The first QTL for APR to YR, QYr.cim- $2 A L$, was located in the interval of DArTSeq markers 3064488_30:T>G and 1106314_11:A>G on chromosome 2AL (Fig. 1E). It explained 5.1 to $8.9 \%$ of the YR variation across all 3 years (Table 4). The second minor APR QTL, QYr.cim$3 D S$, flanked by molecular markers 3021242 and 2243560 on chromosome 3DS (Fig. 1F), explained 3.9 and $4.0 \%$ of YR variation in YR2012 and AYR2012, respectively (Table 4). Both YR resistance QTL were contributed by Kundan. In addition, a minor QTL derived from Avocet, QYr.cim-5AS, was located in the interval of markers 3025355_10:T>C and 1067589 on chromosome 5AS (Fig. 1G). It was detected in YR2013, YR2014, and YRM and explained 4.0 to $5.1 \%$ of YR variation (Table 4 ).

Table 3. Phenotypic correlations for final leaf rust (Ciudad Obregon: LR2013Y and LR2014Y and El Batan: LR2014B) and stripe rust (Toluca: YR2012, YR2013, and YR2014) severities in the Avocet $\times$ Kundan recombinant inbred line population

\begin{tabular}{lccccc}
\hline Experiment & LR2013Y & LR2014Y & LR2014B & YR2012 & YR2013 \\
\hline LR2014Y & $0.83^{* * z}$ & $\ldots$ & $\ldots$ & $\ldots$ & $\ldots$ \\
LR2014B & $0.82^{* *}$ & $0.79^{* *}$ & $\ldots$ & $\ldots$ & $\ldots$ \\
YR2012 & $0.70^{* *}$ & $0.71^{* *}$ & $0.73^{* *}$ & $\ldots$ & $\ldots$ \\
YR2013 & $0.68^{* *}$ & $0.72^{* *}$ & $0.77^{* *}$ & $0.90^{* *}$ & $\ldots$ \\
YR2014 & $0.63^{* *}$ & $0.67^{* *}$ & $0.74^{* *}$ & $0.87^{* *}$ & $0.90^{* *}$ \\
\hline
\end{tabular}

${ }^{\mathrm{z}}$ Two asterisks (**) indicate $P<0.0001$.

Table 2. Estimated number of resistance genes that conferred adult plant resistance to leaf rust (LR) and stripe rust (YR) in 148 Avocet $\times$ Kundan recombinant inbred lines (RIL) based on Mendelian segregation analysis

\begin{tabular}{|c|c|c|c|c|c|c|}
\hline \multirow[b]{2}{*}{ Response } & \multicolumn{6}{|c|}{ Number of RIL } \\
\hline & LR2013Y & LR2014Y & LR2014B & YR2012 & YR2013 & YR2014 \\
\hline HPTS $^{w}$ & 4 & 12 & 11 & 6 & 7 & 6 \\
\hline HPTR $^{x}$ & 3 & 8 & 9 & 5 & 2 & 4 \\
\hline OTHER $^{y}$ & 134 & 121 & 121 & 130 & 132 & 131 \\
\hline Missing & 7 & 7 & 7 & 7 & 7 & 7 \\
\hline Total & 148 & 148 & 148 & 148 & 148 & 148 \\
\hline Genes $(n)$ & 4 & 3 & 3 & 4 & 4 & 4 \\
\hline$P$ value $\mathrm{z}$ & 0.52 & 0.51 & 0.65 & 0.94 & 0.27 & 0.82 \\
\hline
\end{tabular}


Additive interaction between detected resistance loci. The Avocet $\times$ Kundan RIL were divided into 16 and 20 genotypes based on the presence of four LR and five YR APR QTL (Tables 5 and 6). The flanking markers for each QTL were used in determining the presence of parental alleles in the RIL. Three lines in the population possessed all four LR QTL and their mean DS (LRM) was $14.6 \%$ (Table 5). Lines with three QTL combinations showed LRM of 16.0 to $47.8 \%$. Generally, more QTL imparted higher LR resistance in the RIL, although most of the differences in mean severities were not significant for each minor QTL individual (Table 5). Similarly, a mean of YR severity was $24.5 \%$ for the three RILs carrying all five YR resistance QTL, whereas four RILs carrying resistance QTL combination $1 \mathrm{BL}+2 \mathrm{AL}+6 \mathrm{AL}$ showed the lowest YR severities in each environment (Table 6). The pleiotropic APR locus Lr46/ Yr29 played the most important role in conferring resistance to both rusts in this population. Mean LR and YR severity reductions of 28.8 and $33.8 \%$, respectively, were obtained for RILs with Lr46/ Yr29, compared with RILs without it across 3 years (Table 5, 6).

\section{Discussion}

Kundan is an Indian spring wheat cultivar released in 1985, and has remained resistant to LR and YR at the adult plant stage in Mexico and
India. The qualitative and quantitative genetic approaches estimated that there were at least three and four APR genes for LR and YR, respectively, that segregated in the RIL population, which is consistent with the QTL mapping results that identified two co-located resistance QTL, two LR QTL, and three YR QTL in the Avocet $\times$ Kundan population. However, previous studies have reported that qualitative and quantitative methods normally underestimate the number of resistance genes in comparison with the number of QTL detected in mapping population. This may be because of assumptions such as additive effects of polygenes and their equal contributions to phenotypic variation for disease resistance (Basnet et al. 2013; Lan et al. 2014; Wright 1968).

Co-located resistance QTL to $\mathbf{L R}$ and YR. Two co-located resistance QTL, Lr46/Yr29 and QLr.cim-6AL/QYr.cim-6AL, were detected in the Avocet $\times$ Kundan RIL population, which was expected based on high correlation between LR and YR severities ( $r=0.63$ to 0.77 ). The 1BL co-located resistance QTL was identified as the known pleotropic APR gene $L r 46 / Y r 29$ based on the tightly linked marker $c s L V 46 G 22$. Lr46/Yr29, which was first identified in 'Pavon 76' (Singh et al. 1998; William et al. 2003), and provides partial APR to the three rust diseases and powdery mildew (Herrera-Foessel et al. 2014; Lillemo et al. 2008, 2013 ) in wheat. $1 \mathrm{BL}$ is also associated with the LTN gene, Ltn2 (Rosewarne et al. 2006; William et al. 2003). Kundan derived from the

Table 4. Position and effect of quantitative trait loci (QTL) that were detected for adult plant resistance to leaf rust (LR) and stripe rust (YR), area under the disease progress curve (namely, ALR2013Y), and mean of final disease severities over all environments (LRM and YRM) using inclusive composite interval mapping by IciMapping 4.1 using 131 selected recombinant inbred lines from the Avocet $\times$ Kundan population

\begin{tabular}{|c|c|c|c|c|c|c|}
\hline QTL $^{\mathbf{u}}$ & Environment & $\operatorname{Pos}^{v}$ & Marker interval & LOD $^{\mathbf{w}}$ & $\operatorname{PVE}(\%)^{\mathrm{x}}$ & $\operatorname{Add}^{\mathbf{y}}$ \\
\hline \multirow[t]{7}{*}{$L r 46^{\mathrm{z}}$} & LR2013Y & 22 & csLV46G22-1102414 & 26.9 & 49.5 & 14.5 \\
\hline & ALR2013Y & 21 & $1092272-1164928$ & 25.2 & 52.5 & 188.7 \\
\hline & LR2014Y & 21 & $1092272-1164928$ & 24.4 & 57.2 & 18.8 \\
\hline & ALR2014Y & 21 & $1092272-1164928$ & 28 & 61.9 & 72.8 \\
\hline & LR2014B & 21 & $1092272-1164928$ & 23.6 & 64.5 & 15.1 \\
\hline & ALR2014B & 21 & $1092272-1164928$ & 22.4 & 62.2 & 115.3 \\
\hline & LRM & 21 & $1092272-1164928$ & 32.4 & 65.1 & 15.8 \\
\hline \multirow[t]{7}{*}{$\operatorname{Yr} 29$} & YR2012 & 21 & $1092272-1164928$ & 31.8 & 66.1 & 19.3 \\
\hline & AYR2012 & 21 & $1092272-1164928$ & 31.2 & 65.6 & 239.7 \\
\hline & YR2013 & 21 & $1092272-1164928$ & 30.5 & 57 & 18 \\
\hline & AYR2013 & 21 & $1092272-1164928$ & 28.2 & 55 & 96.9 \\
\hline & YR2014 & 21 & $1092272-1164928$ & 24.8 & 49.2 & 16.1 \\
\hline & AYR2014 & 21 & $1092272-1164928$ & 27.5 & 53.9 & 114.1 \\
\hline & YRM & 21 & $1092272-1164928$ & 36.5 & 63.1 & 18 \\
\hline \multirow{4}{*}{ QLr.cim-6AL } & LR2013Y & 22 & 1021176_60:T>G-2259361 & 2.4 & 8.5 & -3.7 \\
\hline & LR2014Y & 28 & 1076263_18:C>T-1101578 & 3.2 & 10.9 & -6.6 \\
\hline & YR2013 & 22 & 1021176_60:T>G-2259361 & 3.5 & 12.4 & -8.1 \\
\hline & AYR2013 & 22 & 1021176_60:T>G-2259361 & 3.5 & 12.6 & -52 \\
\hline \multirow[t]{4}{*}{ QYr.cim-6AL } & YR2014 & 22 & 1021176_60:T>G-2259361 & 2.9 & 10.2 & -7.9 \\
\hline & AYR2014 & 28 & 1076263_18:C>T-1101578 & 2.5 & 9 & -49 \\
\hline & YRM & 22 & 1021176_60:T>G-2259361 & 2.6 & 9.3 & -7.7 \\
\hline & LR2013Y & 459 & 1218181-1081780_35:C>T & 3.5 & 4.1 & -4.4 \\
\hline \multirow[t]{2}{*}{ QLr.cim- $2 B L$} & LR2014Y & 455 & $1237388-1265455$ & 3.6 & 5.6 & -5.9 \\
\hline & ALR2014Y & 455 & $1237388-1265455$ & 3.7 & 4.9 & -20.6 \\
\hline \multirow[t]{2}{*}{ QLr.cim-2DS } & LR2013Y & 1 & $1020115-1242814$ & 6.6 & 7.8 & 5.7 \\
\hline & ALR2013Y & 1 & $1020115-1242814$ & 4.5 & 6.4 & 65.7 \\
\hline \multirow{6}{*}{ QYr.cim- $2 A L$} & YR2012 & 87 & 1089750_12:A>G-1106314_11:A>G & 7.2 & 8.9 & 7.1 \\
\hline & AYR2012 & 87 & 1089750_12:A>G-1106314_11:A>G & 6.4 & 8.1 & 83.9 \\
\hline & AYR2013 & 84 & 998788_19:T>C-3959527 & 6.6 & 8.4 & 37.8 \\
\hline & YR2014 & 86 & 3064488_30:T>G-1089750_12:A>G & 4 & 5.1 & 5.2 \\
\hline & AYR2014 & 86 & 3064488_30:T>G-1089750_12:A>G & 4.8 & 6 & 37.8 \\
\hline & YRM & 87 & 1089750_12:A>G-1106314_11:A>G & 6 & 5.5 & 5.3 \\
\hline \multirow[t]{2}{*}{ QYr.cim-3DS } & YR2012 & 55 & $3021242-2243560$ & 3.4 & 4 & 4.8 \\
\hline & AYR2012 & 55 & $3021242-2243560$ & 3.3 & 3.9 & 59.5 \\
\hline \multirow{3}{*}{ QYr.cim-5AS } & YR2013 & 168 & 3025355_10:T>C-2262017_48:A>G & 3.8 & 4.1 & -4.9 \\
\hline & YR2014 & 174 & $3938058-1067589$ & 4 & 5.1 & -5.2 \\
\hline & YRM & 168 & 3025355_10:T>C-2262017_48:A>G & 4.4 & 4 & -4.7 \\
\hline
\end{tabular}

u QTL that extend across single one-log support confidence intervals were assigned the same symbol.

${ }^{v}$ Peak position in centimorgans from the first linked marker of the relevant linkage group.

${ }^{\text {w }}$ Logarithm of odds score based on 1,000 permutations.

x Percentage of phenotypic variance explained by the QTL.

y Additive effect of each QTL on disease severities.

z $\mathrm{Y}$ and $\mathrm{B}$ indicate Ciudad Obregon and El Batan, respectively. 
cross of Tanori 71/NP 890, whereas 'Tanori 71' has the pedigree Sonora 64//Ciano-67/Inia 66 and 'NP 890' was C281//C281/Gaza. All of these lines carried the positive allele of $L r 46 / Y r 29$ based on the closely linked marker $c s L V 46 G 22$, indicating that all parents possibly possessed the resistance gene or, more likely, the lack of diagnostic ability of the marker, because 'Sonora 64' and 'Inia 66' are known to be susceptible to LR. $L r 46 / Y r 29$ is known to be present in many wheat lines developed at CIMMYT, because it continues to play a key role in breeding for durable rust resistance against LR and YR (Basnet et al. 2013; Lan et al. 2014; Lillemo et al. 2008; Ren et al. 2012b; Rosewarne et al. 2006; Singh et al. 2011; Yang et al. 2013). Previous studies reported that Lr46/Yr29 explained 16 to $55 \%$ and 11 to $43 \%$ of total phenotypic variation for LR and YR, respectively, and the additive effect of $L r 46 / Y r 29$ was much less in the presence of Lr34/Yrl8 or Yr54 (Basnet et al. 2014; Lan et al. 2014; Lillemo et al. 2008; Rosewarne et al. 2012). In our study, $L r 46 / Y r 29$ explained 49.5 to $65.1 \%$ and 49.2 to $66.1 \%$ of LR and YR severity variations, respectively. This was in accordance with the previously observed results that this gene reduced LR and YR severities up to 40 to $50 \%$ when it was present alone (Lan et al. 2014; Rosewarne et al. 2012; Singh et al. 1998), indicating that a single APR gene does not provide acceptable levels of resistance. Therefore, it is necessary to combine this gene with other APR genes to achieve adequate levels of resistance in the field as in Kundan.

The second co-located resistance QTL, QLr.cim-6AL/QYr.cim-6AL, was found to be inherited from the susceptible parent Avocet. This QTL was located on chromosome arm 6AL and flanked by DArT-seq markers 1021176_60 and 2259361. These markers have been mapped in the vicinity of DArT-array markers $w P t-4229, w P t-6829$, and $w P t$ 8954 and SSR markers Xgwm999 and Xgwm1089 at the distal end of chromosome 6AL (Marone et al. 2012). Two LR APR QTL, QLr.cim-6AL (William et al. 2006) and QLr.Hbau-6AL (Zhang et al. 2009), have been reported on chromosome 6AL from Avocet. Both of them were located in the middle of chromosome 6AL and linked to SSR marker Xgwm617 within genetic distances of 11.5 and 16.0 cM, respectively. William et al. (2006) also mapped a YR resistance QTL QYr6A.2 in the same region in Avocet. It was flanked by SSR markers Xgwm617 and Xgwm427. In addition, Lillemo et al. (2008) detected a YR resistance QTL close to the centromere on the long arm of chromosome 6A, which was linked to Xbarc3 with a genetic distance of $3.3 \mathrm{cM}$ and explained $14 \%$ of the YR variance in Avocet. These QTL were located in the same genomic region as $Q L$ r.cim- $6 A L Q Y$ r.cim- $6 A L$ based on an integrated linkage map of diploid wheat (Singh et al. 2007). Although this locus was not detected in all tested environments, it is likely to be a useful locus in combination with other genes.

APR QTL for LR. A QTL for APR to LR in Avocet, QLr.cim-2BL, was mapped to the proximal region of chromosome arm $2 \mathrm{BL}$ and flanked by DArT-Seq markers 1237388 and 1265455 . These markers have been mapped near DArT-array marker $w P t-5736$ and SSR marker Xbarc101 (Marone et al. 2012). Two LR resistance QTL, QLr.sfrs-2BL and QLr.osu-2B, were located on chromosome 2BL (Messmer et al. 2000; Xu et al. 2005). QLr.sfrs-2BL was derived from Swiss winter spelt (T. spelta L.) 'Oberkulmer', whereas $Q L$ r.osu- $2 B$ derived from common wheat 'CI 13227' was located on the centromere region of chromosome
A 1 BL

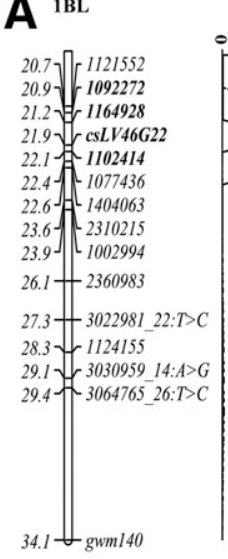

$D_{2 \mathrm{DS}}$

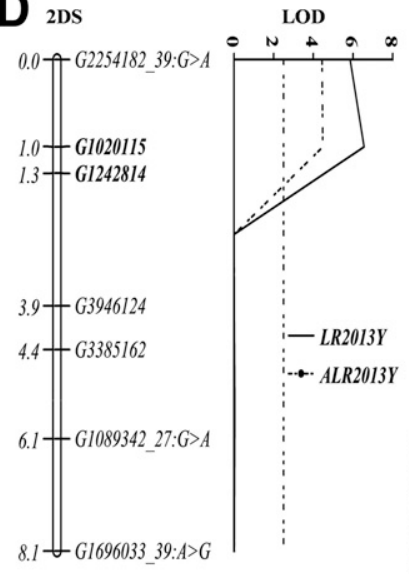

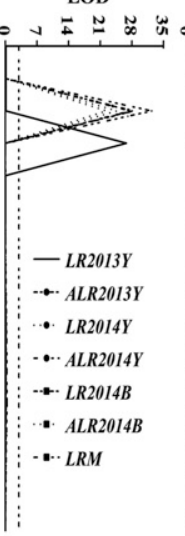
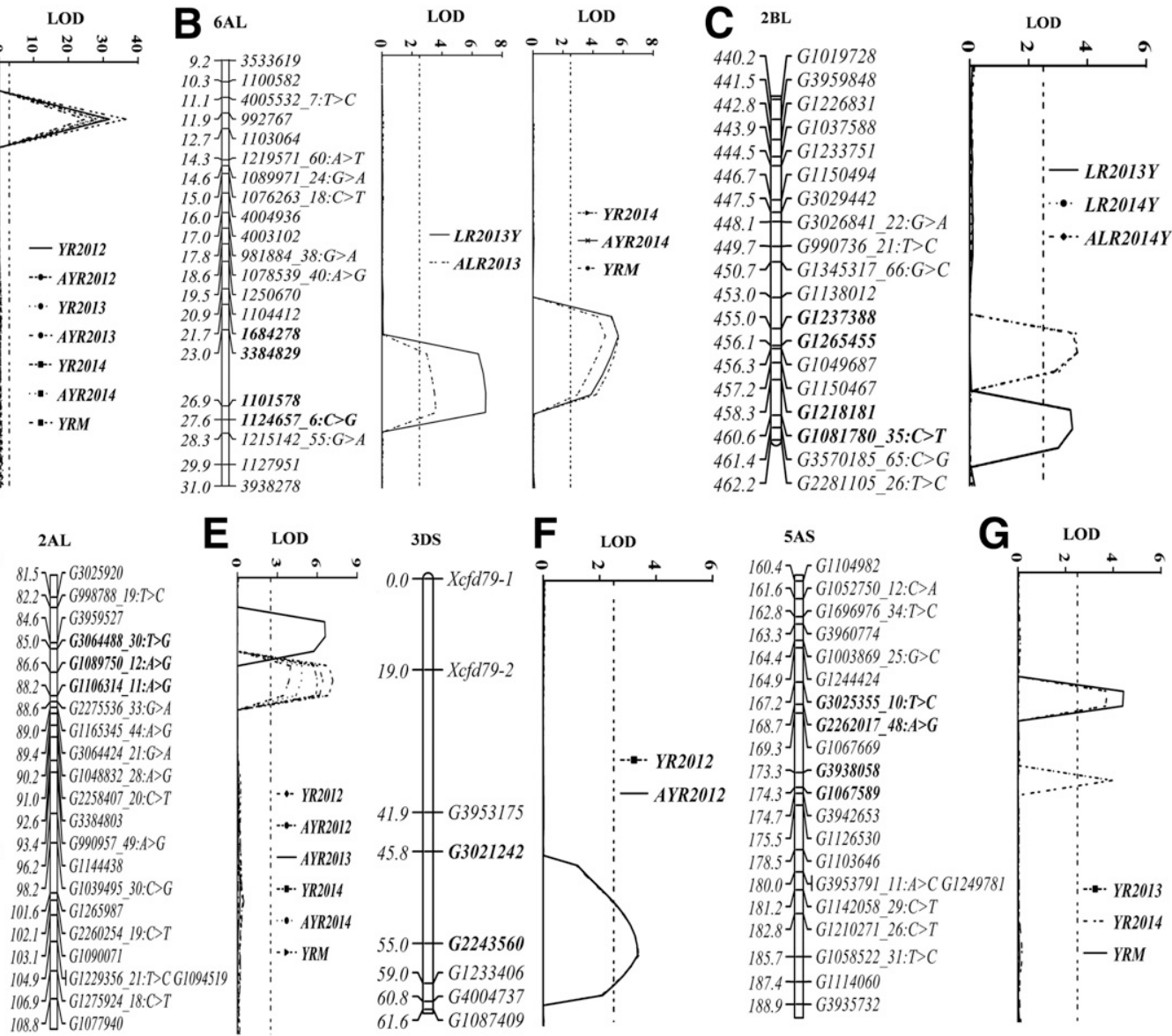

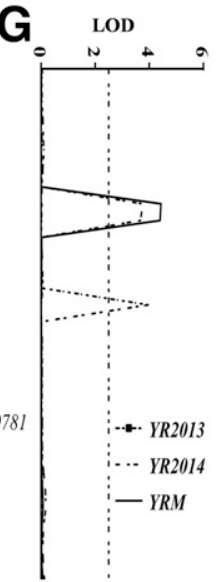

Fig. 1. Likelihood plots of quantitative trait loci for adult plant resistance to leaf rust (LR) on chromosomes $A, 1 B L ; B, 6 A L ; C, 2 B L ;$ and $D$, 2DS; and to stripe rust (YR) on chromosomes A, 1BL; B, 6AL; E, 3DS; F, 2AL; and G, 5AS identified by lciMapping 4.1 in the Avocet $\times$ Kundan recombinant inbred line population. The significant logarithm of odds threshold was detected based on 1,000 permutations. Positions (in centimorgans) of cumulated genetic distances of linkage group along chromosomes are shown on the left axes and for molecular markers on the right. LR2013Y, LR2014Y, and LR2014B = final LR severity at Ciudad Obregon during 2012-13 and 2013-14 and El Batan in 2014, respectively; YR2012, YR2013, and YR2014 = final YR severity in Toluca in 2012, 2013, and 2014, respectively; ALR2013Y, ALR2014Y, ALR2014B, AYR2012, AYR2013, and AYR2014 = area under the disease progress curve for LR in Obregon 2012-13 and 2013-14 and El Batan 2014, and for YR in 2012, 2013, and 2014 in Toluca, respectively; LRM and YRM = the mean of final disease severity over LR2013Y, LR2014Y, and LR2014B and over YR2012, YR2013, and YR2014 for LR and YR, respectively. 
2B and linked to SSR markers Xbarc18, Xbarc167, and Xwmc314, with a genetic distance around $20 \mathrm{cM}$ away from $Q L$ r.cim- $2 B L$ based on the wheat consensus map (Somers et al. 2004). Therefore, it is likely that QLr.cim-2BL is a new LR resistance locus in common wheat.

The second APR QTL for LR derived from Kundan, QLr.cim-2DS, was located in the interval of markers 1020115 and 1242814 on the distal region of chromosome 2DS-5, based on the DArT physical map (http://www.cerealsdb.uk.net/cerealgenomics/CerealsDB/dart_ chromosome.php?chromosome=2D). These markers are closely linked to DArT-array marker $w P t-2014$ and SSR markers Xcfd51, $X c f d 56, X c f d 36$, and Xbarc124 in this genomic region (Francki et al. 2009). Zhang et al. (2009) and Xu et al. (2005) detected two minor LR QTL, QLr.hbau-2DS and QLrid.osu-2DS, respectively, in chromosome 2DS. QLr.hbau-2DS was linked to Xbarc124 and Xgwm296 and explained 12.2 to $12.5 \%$ of the LR severity in CIMMYT 'Saar' spring wheat. QLrid.osu-2DS derived from common wheat CI 13227 was located in the interval of SSR marker Xgwm261 and AFLP marker XGCTG.CGCT118 within $12 \mathrm{cM}$ of QLr.cim-2DS based on the wheat consensus map (Somers et al. 2004; Xu et al. 2005). In addition, two minor LR QTL, QLr.cim-2DS and QLr.sfr-2DS, were also mapped in this region in chromosome 2DS in common wheat Avocet and 'Forno', respectively (Rosewarne et al. 2012; Schnurbusch et al.
2004). Therefore, QLr.cim-2DS might be the same LR resistance locus as QTL in Saar, CI 13227, and Forno but different from the QTL in Avocet because we would not have detected this locus in the present population if they are the same.

APR QTL for YR. The first QTL for APR to YR in Kundan, QYr.cim-2AL, was located in the interval of DArT-seq markers 3064488_30:T> $G$ and 1106314_11:A $>G$ near the centromere of chromosome $2 \mathrm{AL}$. These markers were also mapped in the vicinity of DArT-array marker $w P t-7306$ and SSR markers Xbarc220 and Xgwm71 (Zhang et al. 2008). Two minor YR resistance QTL, $Q Y r$. inra-2AL (Dedryver et al. 2009) and QYR2 (Boukhatem et al. 2002), were mapped on chromosome $2 \mathrm{AL}$ in common wheat 'Récital' and 'Camp Remy', respectively, and closely linked to the same SSR marker Xgwm382 in the distal region of $2 \mathrm{AL}$ (Boukhatem et al. 2002; Dedryver et al. 2009). However, $Q Y$ r.cim-2AL was near the centromere of chromosome $2 \mathrm{AL}$ within $86 \mathrm{cM}$ of $Q Y$ r.inra- $2 A L$ and QYR2 based on the wheat consensus map (Francki et al. 2009; Somers et al. 2004), indicating the novelty of this consistent YR resistance locus in Kundan.

The second minor QTL, QYr.cim-3DS, was located on the short arm of chromosome 3D and was effective only in 2012 at Toluca. QYr. inra-3DS was located in the interval of SSR markers Xbarc125 and

Table 5. Mean leaf rust (LR) severities of Avocet $\times$ Kundan recombinant inbred lines (RIL) carrying different quantitative trait loci (QTL) combinations ${ }^{\mathrm{y}}$

\begin{tabular}{|c|c|c|c|c|c|}
\hline QTL combination & RIL (n) & LR2013Y & LR2014Y & LR2014B & $\mathbf{L R M}^{\mathbf{z}}$ \\
\hline None & 15 & $60.3 \mathrm{a}$ & $73.0 \mathrm{a}$ & $55.0 \mathrm{a}$ & $63.0 \mathrm{a}$ \\
\hline $6 \mathrm{AL}$ & 3 & $60.0 \mathrm{a}$ & $66.7 \mathrm{a}$ & $50.0 \mathrm{a}$ & $58.9 \mathrm{a}$ \\
\hline $2 \mathrm{BL}$ & 8 & $59.4 \mathrm{a}$ & $60.0 \mathrm{~b}$ & $50.0 \mathrm{a}$ & $56.5 \mathrm{a}$ \\
\hline 2DS & 5 & $56.0 \mathrm{a}$ & $68.0 \mathrm{a}$ & $50.0 \mathrm{a}$ & $58.0 \mathrm{a}$ \\
\hline $2 \mathrm{BL}+2 \mathrm{DS}$ & 11 & $46.4 \mathrm{~b}$ & $69.1 \mathrm{a}$ & $48.2 \mathrm{a}$ & $54.6 \mathrm{~b}$ \\
\hline $2 \mathrm{BL}+6 \mathrm{AL}$ & 2 & $40.0 \mathrm{~b}$ & $60.0 \mathrm{~b}$ & $40.0 \mathrm{~b}$ & $46.7 \mathrm{~b}$ \\
\hline $2 \mathrm{BL}+2 \mathrm{DS}+6 \mathrm{AL}$ & 3 & $36.7 \mathrm{~b}$ & $66.7 \mathrm{a}$ & $40.0 \mathrm{~b}$ & $47.8 \mathrm{~b}$ \\
\hline $2 \mathrm{DS}+6 \mathrm{AL}$ & 2 & $35.0 \mathrm{~b}$ & $75.0 \mathrm{a}$ & $35.0 \mathrm{~b}$ & $48.4 \mathrm{~b}$ \\
\hline $1 \mathrm{BL}+6 \mathrm{AL}$ & 6 & $33.3 \mathrm{c}$ & $49.2 \mathrm{c}$ & $28.3 \mathrm{c}$ & $36.9 \mathrm{~b}$ \\
\hline $1 \mathrm{BL}$ & 6 & $30.0 \mathrm{c}$ & $41.3 \mathrm{c}$ & $31.3 \mathrm{c}$ & $34.2 \mathrm{c}$ \\
\hline $1 \mathrm{BL}+2 \mathrm{BL}$ & 9 & $24.4 \mathrm{c}$ & $36.1 \mathrm{c}$ & $30.0 \mathrm{c}$ & $30.2 \mathrm{c}$ \\
\hline $1 \mathrm{BL}+2 \mathrm{DS}$ & 16 & $23.8 \mathrm{c}$ & $35.9 \mathrm{c}$ & $22.6 \mathrm{c}$ & $27.4 \mathrm{c}$ \\
\hline $1 \mathrm{BL}+2 \mathrm{DS}+6 \mathrm{AL}$ & 6 & $15.8 \mathrm{~d}$ & $30.8 \mathrm{~d}$ & $15.8 \mathrm{~d}$ & $20.8 \mathrm{~d}$ \\
\hline $1 \mathrm{BL}+2 \mathrm{BL}+2 \mathrm{DS}$ & 10 & $12.5 \mathrm{~d}$ & $26.6 \mathrm{~d}$ & $15.1 \mathrm{~d}$ & $18.1 \mathrm{e}$ \\
\hline $1 \mathrm{BL}+2 \mathrm{BL}+6 \mathrm{AL}$ & 4 & $13.8 \mathrm{~d}$ & $23.1 \mathrm{~d}$ & $11.3 \mathrm{e}$ & $16.0 \mathrm{e}$ \\
\hline $1 \mathrm{BL}+2 \mathrm{BL}+2 \mathrm{DS}+6 \mathrm{AL}$ & 3 & $12.7 \mathrm{~d}$ & $25.8 \mathrm{~d}$ & $5.3 \mathrm{e}$ & $14.6 \mathrm{e}$ \\
\hline
\end{tabular}

y Different letters within a column following the mean indicate significant differences based on a $t$ test $(P<0.05)$.

${ }^{\mathrm{z}}$ Mean of final disease severities over all environments.

Table 6. Mean stripe rust (YR) severities of Avocet $\times$ Kundan recombinant inbred lines (RIL) carrying different quantitative trait loci (QTL) combinations ${ }^{\mathrm{y}}$

\begin{tabular}{|c|c|c|c|c|c|}
\hline QTL combination & RIL (n) & YR2012 & YR2013 & YR2014 & $\mathbf{Y R M}^{\mathbf{Z}}$ \\
\hline None & 9 & $78.3 \mathrm{a}$ & $86.1 \mathrm{a}$ & $85.0 \mathrm{a}$ & $83.2 \mathrm{a}$ \\
\hline $3 \mathrm{DS}+6 \mathrm{AL}$ & 2 & $72.5 \mathrm{a}$ & $75.0 \mathrm{a}$ & $85.0 \mathrm{a}$ & $77.5 \mathrm{a}$ \\
\hline $5 \mathrm{AS}$ & 2 & $62.5 \mathrm{~b}$ & $75.0 \mathrm{a}$ & $67.5 \mathrm{a}$ & $68.4 \mathrm{~b}$ \\
\hline $2 \mathrm{AL}+5 \mathrm{AS}$ & 6 & $62.5 \mathrm{~b}$ & $71.7 \mathrm{~b}$ & $74.0 \mathrm{a}$ & $68.6 \mathrm{~b}$ \\
\hline $2 \mathrm{AL}+3 \mathrm{DS}$ & 3 & $60.0 \mathrm{~b}$ & $68.3 \mathrm{~b}$ & $62.5 \mathrm{~b}$ & $63.9 \mathrm{~b}$ \\
\hline $2 \mathrm{AL}+6 \mathrm{AL}$ & 4 & $52.5 \mathrm{~b}$ & $62.5 \mathrm{~b}$ & $62.5 \mathrm{~b}$ & $59.2 \mathrm{~b}$ \\
\hline $2 \mathrm{AL}$ & 2 & $50.0 \mathrm{~b}$ & $55.0 \mathrm{~b}$ & $65.0 \mathrm{a}$ & $56.7 \mathrm{~b}$ \\
\hline $2 \mathrm{AL}+3 \mathrm{DS}+5 \mathrm{AS}$ & 9 & $43.3 \mathrm{c}$ & $55.0 \mathrm{~b}$ & $58.1 \mathrm{~b}$ & $52.2 \mathrm{c}$ \\
\hline $1 \mathrm{BL}$ & 9 & $37.8 \mathrm{c}$ & $52.8 \mathrm{c}$ & $57.8 \mathrm{~b}$ & $49.4 \mathrm{c}$ \\
\hline $1 \mathrm{BL}+6 \mathrm{AL}$ & 6 & $29.2 \mathrm{~d}$ & $35.8 \mathrm{c}$ & $46.7 \mathrm{c}$ & $37.2 \mathrm{~d}$ \\
\hline $1 \mathrm{BL}+2 \mathrm{AL}$ & 3 & $26.7 \mathrm{~d}$ & $53.3 \mathrm{c}$ & $56.7 \mathrm{~b}$ & $45.6 \mathrm{c}$ \\
\hline $1 \mathrm{BL}+5 \mathrm{AS}$ & 2 & $25.0 \mathrm{~d}$ & $40.0 \mathrm{c}$ & $45.0 \mathrm{c}$ & $36.7 \mathrm{~d}$ \\
\hline $1 \mathrm{BL}+3 \mathrm{DS}$ & 4 & $20.0 \mathrm{~d}$ & $37.5 \mathrm{c}$ & $45.0 \mathrm{c}$ & $34.2 \mathrm{~d}$ \\
\hline $1 \mathrm{BL}+2 \mathrm{AL}+3 \mathrm{DS}$ & 6 & $18.0 \mathrm{~d}$ & $27.9 \mathrm{~d}$ & $38.8 \mathrm{c}$ & $28.2 \mathrm{~d}$ \\
\hline $1 \mathrm{BL}+2 \mathrm{AL}+5 \mathrm{AS}$ & 4 & $17.5 \mathrm{~d}$ & $22.5 \mathrm{~d}$ & $24.5 \mathrm{~d}$ & $21.5 \mathrm{e}$ \\
\hline $1 \mathrm{BL}+2 \mathrm{AL}+3 \mathrm{DS}+5 \mathrm{AS}+6 \mathrm{AL}$ & 3 & $16.7 \mathrm{~d}$ & $23.3 \mathrm{~d}$ & $33.3 \mathrm{c}$ & $24.5 \mathrm{~d}$ \\
\hline $1 \mathrm{BL}+3 \mathrm{DS}+5 \mathrm{AS}$ & 2 & $12.5 \mathrm{~d}$ & $25.0 \mathrm{~d}$ & $35.0 \mathrm{c}$ & $24.2 \mathrm{~d}$ \\
\hline $1 \mathrm{BL}+3 \mathrm{DS}+5 \mathrm{AS}+6 \mathrm{AL}$ & 2 & $12.5 \mathrm{~d}$ & $30.0 \mathrm{~d}$ & $21.5 \mathrm{~d}$ & $21.3 \mathrm{e}$ \\
\hline $1 \mathrm{BL}+2 \mathrm{AL}+6 \mathrm{AL}$ & 4 & $9.0 \mathrm{e}$ & $20.0 \mathrm{~d}$ & $18.8 \mathrm{~d}$ & $15.9 \mathrm{e}$ \\
\hline
\end{tabular}

${ }^{\mathrm{y}}$ Different letters within a column following the mean indicate significant differences based on a $t$ test $(P<0.05)$.

${ }^{\mathrm{z}}$ Mean of final disease severities over all environments. 
Xgwm456a on chromosome 3DS and explained 4.7 to $8.3 \%$ of the phenotypic variation in common wheat Récital (Dedryver et al. 2009). QYr.cim-3DL was flanked by SSR markers $\mathrm{Xgdm} 8$ and $\mathrm{Xgdm} 128$ and explained 3.6 to $13.5 \%$ of the YR variation in 'Chapio' (Yang et al. 2013). QYr.Tam-3D was located in the interval of markers $w P t-672034$ and Xbarc 125 and explained 4 to $5 \%$ of the YR variation in CIMMYT line 'Quaiu 3' (Basnet et al. 2014). The genetic distance between QYr.cim-3DL, QYr.inra-3DS, and QYr.Tam-3D was estimated to be about 15 to $19 \mathrm{cM}$ based on the consensus map (Francki et al. 2009; Somers et al. 2004). However, we cannot rule out the relationship between QYr.cim-3DS and QTL in Récital, Chapio, and Quaiu 3 due to the unavailable genetic map of flanked DArT-seq markers 3021242 and 2243560. Despite the observed minor effect of these loci on YR and their inability to be detected in all environments, they possibly play an important role in CIMMYT wheat germplasms for breeding resistant lines.

QTL QYr.cim-5AS, derived from Avocet had significant effects on YR in YR2013, YR2014, and YRM. It was located in the interval of DArT-seq markers 3025355, 2262017, 3938058, and 1067589 and mapped near the DArT-array markers wPt-4131,wPt-0605, and $w P t-742925$ and SSR markers Xcfa2104, Xbarc186, and Xwmc418 based on a DArT and SSR consensus map from a multiparent advanced generation intercross wheat population (Huang et al. 2012). Five YR resistance QTL were mapped in T. boeoticum and wheat lines 'PAU5088' (Chhuneja et al. 2008), 'Opata 85' (Boukhatem et al. 2002), 'Pingyuan 50' (Lan et al. 2010), 'Pastor' (Rosewarne et al. 2012), and 'Shanghai 3' × 'Catbird' (Ren et al. 2012a) in chromosome 5AL. QYrtb.pau-5A from T. boeoticum mapped on 5AL in the 8.9-cM interval between Xbarc151 and Xcfd12. The Opata 85 QTL was observed in seedling tests. The Pingyuan 50 QTL was flanked by Xwmc410 and Xbarc261 and located in the same region as QTL in Pastor and Shanghai $3 \times$ Catbird. Because no other YR resistance QTL has been reported on chromosome 5AS thus far, it is possible that QYr.cim-5AS is a new APR QTL to YR.

Additive effect between detected QTL. The mean LR severities of RIL combining Lr46/Yr29 with two or three LR resistance QTL were significantly lower than the lines with $L r 46 / Y r 29$ alone. Similar additive interaction between QTL was also detected in the resistance to YR. Lan et al. (2014) also analyzed the additive interaction between Lr46/Yr29 and other detected QTL for LR and YR resistance in the Avocet $\times$ 'Francolin 1' population and concluded that there were no significant additive interactions between minor QTL in the background of strong-effect gene $L r 46 / Y r 29$ for LR. However, significant YR reductions were observed with RIL carrying $L r 46 / Y r 29$ and other two or more minor QTL, together with the exceptions of QYr.cim-3DS and QLr.cim-6AL/QYr.cim-6AL. Of the 70 and $80 \%$ of the phenotypic variations explained by detected LR and YR QTL, respectively, in the present population, $L r 46 / Y r 29$ alone contributed a large proportion of the variation (65.1 and $66.1 \%$, respectively). As far as the resistance loci derived from the susceptible parent Avocet are concerned, two RILs carrying both resistance loci (2BL and 6AL) showed lower LR severity (40 to $60 \%$ ) than the susceptible parent Avocet (90 to 100\%). This difference could be due to the other undetected minor LR resistance QTL in the population.

Although the durability of effective race-specific resistance genes can be prolonged by using gene combinations, an alternative approach is to develop varieties that possess APR based on combinations of pleiotropic and other minor, slow-rusting genes. Kundan, with good agronomic characteristics and a high level of APR to LR and YR, can be used in wheat breeding programs as a source of slow-rusting APR genes. Resistance loci Lr46/Yr29, QLr.cim-2DS, QYr.cim-2AL, and QYr.cim-3DS were derived from Kundan. The genetic interval between flanking markers of these QTL ranged from 0.3 to $4.2 \mathrm{cM}$. With the availability of defined molecular markers for each resistance locus, pyramiding the APR gene from the single resistance source Kundan should be easier and more efficient in the future. However, all of the closely linked markers are DArT-seq and need to be converted into breeder-friendly kompetitive allele specific PCR (KASP) markers and validated in a broader set of germplasm before being used in marker-assisted selection for improving resistance to LR and YR in wheat breeding.

\section{Acknowledgments}

This work was supported by the Australian Grains Research and Development Corporation, Australian Cereal Rust Control Program, National Natural Science Foundation of China (31301309), and International Joint Research Project of Sichuan Province (2014HH0052). We thank J. Mollins for technical editing.

\section{Literature Cited}

Basnet, B. R., Singh, R. P., Herrera-Foessel, S. A., Ibrahim, A. M. H., Huerta-Espino, J., Calvo-Salazar, V., and Rudd, J. 2013. Genetic analysis of adult plant resistance to yellow rust and leaf rust in common spring wheat Quaiu 3. Plant Dis. 97:728-736.

Basnet, B. R., Singh, R. P., Ibrahim, A. M. H., Herrera-Foessel, S. A., HuertaEspino, J., Lan, C. X., and Rudd, J. C. 2014. Characterization of Yr54 and other genes associated with adult plant resistance to yellow rust and leaf rust in common wheat Quaiu 3. Mol. Breed. 33:385-399.

Bjarko, M. E., and Line, R. F. 1988. Heritability and number of genes controlling leaf rust resistance in four cultivars of wheat. Phytopathology 78:457-461.

Boukhatem, N., Baret, P. V., Mingeot, D., and Jacquemin, J. M. 2002. Quantitative trait loci for resistance against yellow rust in two wheat derived recombinant inbred line populations. Theor. Appl. Genet. 104:111-118.

Chen, X. M. 2005. Epidemiology and control of stripe rust (Puccinia striiformis f. sp. tritici) on wheat. Can. J. Plant Pathol. 27:314-337.

Chen, X. M. 2013. Review article: High-temperature adult-plant resistance, key for sustainable control of stripe rust. Am. J. Plant Sci. 4:608-627.

Chen, X. M. 2014. Integration of cultivar resistance and fungicide application for control of wheat stripe rust. Can. J. Plant Pathol. 36:311-326.

Chhuneja, P., Kaur, S., Garg, T., Ghai, M., Kaur, S., Prashar, M., Bains, N. S., Goel, R. K., Keller, B., Dhaliwal, H. S., and Singh, K. 2008. Mapping of adult plant stripe rust resistance genes in diploid A genome wheat species and their transfer to bread wheat. Theor. Appl. Genet. 116:313-324.

CIMMYT. 2005. Laboratory Protocols: CIMMYT Applied Molecular Genetics Laboratory, 3rd ed. CIMMYT, México D.F., Mexico.

Collard, B. C., and Mackill, D. J. 2008. Marker-assisted selection, an approach for precision plant breeding in the twenty-first century. Philos. Trans. R. Soc. B Biol. Sci. 363:557-572.

Dedryver, F., Paillard, S., Mallard, S., Robert, O., Trottet, M., Nègre, S., Verplancke, G., and Jahier, J. 2009. Characterization of genetic components involved in durable resistance to stripe rust in the bread wheat 'Renan'. Phytopathology 99:968-973.

Dhanda, S. S., and Sethi, G. S. 2002. Tolerance to drought stress among selected Indian wheat cultivars. J. Agric. Sci. 139:319-326.

Elshire, R. J., Glaubitz, J. C., Sun, Q., Poland, J. A., Kawamoto, K., Buckler, E. S., and Mitchell, S. E. 2011. A robust, simple genotyping-by-sequencing (GBS) approach for high diversity species. PLoS One 6:e19379.

Federer, W. T. 1956. Augment (or Hoonuiaku) designs. Hawaii. Plant Rec. 55 191-208.

Federer, W. T. 1961. Augmented designs with one-way elimination of heterogeneity. Biometrics 20:540-552.

Francki, M. G., Walker, E., Crawford, A. C., Broughton, S., Ohm, H. W., Barclay, I., Wilson, R. E., and McLean, R. 2009. Comparison of genetic and cytogenetic maps of hexaploid wheat (Triticum aestivum L.) using SSR and DArT markers. Mol. Genet. Genomics 281:181-191.

Grewal, S., Kharb, P., Malik, R., Jain, S., and Jain, R. K. 2007. Assessment of genetic diversity among some Indian wheat cultivars using random amplified polymorphic DNA (RAPD) markers. Indian J. Biotechnol. 6:18-23.

Helguera, M., Khan, I. A., Kolmer, J., Lijavetzky, D., Li, Z. Q., and Dubcovsky, J. 2003. PCR assays for the Lr37-Yr17-Sr38 cluster of rust resistance genes and their use to develop isogenic hard red spring wheat lines. Crop Sci. 43:1839-1847.

Herrera-Foessel, S. A., Lagudah, E. S., Huerta-Espino, J., Hayden, M. J., Bariana, H. S., Singh, D., and Singh, R. P. 2010. New slow rusting leaf rust and stripe rust resistance genes $\operatorname{Lr} 67$ and $\mathrm{Yr} 46$ in wheat are pleiotropic or closely linked. Theor. Appl. Genet. 122:239-249.

Herrera-Foessel, S. A., Singh, R. P., Huerta-Espino, J., Rosewarne, G. M., Periyannan, S. K., Viccars, L., Calvo-Salazar, V., Lan, C. X., and Lagudah, E. S. 2012. Lr68: A new gene conferring slow rusting resistance to leaf rust in wheat. Theor. Appl. Genet. 124:1475-1486.

Herrera-Foessel, S. A., Singh, R. P., Lillemo, M., Huerta-Espino, J., Bhavani, S. Singh, S., Lan, C. X., Calvo-Salazar, V., and Lagudah, E. S. 2014. Lr67/Yr46 confers adult plant resistance to stem rust and powdery mildew in wheat. Theor. Appl. Genet. 127:781-789.

Huang, B. E., George, A. W., Forrest, K. L., Kilian, A., Hayden, M. J., Morell, M. K., and Cavanagh, C. R. 2012. A multiparent advanced generation inter-cross population for genetic analysis in wheat. Plant Biotechnol. J. 10:826-839.

Huerta-Espino, J., Singh, R. P., Germán, S., McCallum, B. D., Park, R. F., Chen, W. Q., Bhardwaj, S. C., and Goyeau, H. 2011. Global status of wheat leaf rust caused by Puccinia triticina. Euphytica 179:143-160.

Khan, M., Bukhari, A., Dar, Z., and Rizvi, S. 2013. Status and strategies in breeding for rust resistance in wheat. Agric. Sci. 4:292-301.

Knott, D. R., and Padidam, M. 1988. Inheritance of resistance to stem rust in six wheat lines having adult plant resistance. Genome 30:283-288.

Lagudah, E. S. 2011. Molecular genetics of race non-specific rust resistance in wheat. Euphytica 179:81-91. 
Lan, C. X., Liang, S. S., Zhou, X. C., Zhou, G., Lu, Q. L., Xia, X. C., and He, Z. H. 2010. Identification of genomic regions controlling adult-plant stripe rust resistance in Chinese landrace Pingyuan 50 through bulked segregant analysis. Phytopathology 100:313-318.

Lan, C. X., Rosewarne, G. M., Singh, R. P., Herrera-Foessel, S. A., Huerta-Espino, J., Basnet, B. R., Zhang, Y., and Yang, E. 2014. QTL characterization of resistance to leaf rust and stripe rust in the spring wheat line Francolin\#1. Mol. Breed. 34:789-803.

Li, Z. F., Lan, C. X., He, Z. H., Singh, R. P., Rosewarne, G. M., Chen, X. M., and Xia, X. C. 2014. Overview and application of QTL for adult plant resistance to leaf rust and powdery mildew in wheat. Crop Sci. 54:1907-1925.

Lillemo, M., Asalf, B., Singh, R. P., Huerta-Espino, J., Chen, X. M., He, Z. H., and Bjørnstad, A. 2008. The adult plant rust resistance loci $\mathrm{Lr} 34 / \mathrm{Yr} 18$ and $\mathrm{Lr}$ 46/ Yr29 are important determinants of partial resistance to powdery mildew in bread wheat line Saar. Theor. Appl. Genet. 116:1155-1166.

Lillemo, M., Joshi, A. K., Prasad, R., Chand, R., and Singh, R. P. 2013. QTL for spot blotch resistance in bread wheat line Saar co-locate to the biotrophic disease resistance loci Lr34 and Lr46. Theor. Appl. Genet. 126:711-719.

Lin, F., and Chen, X. M. 2007. Genetics and molecular mapping of genes for racespecific all-stage resistance and non-race-specific high temperature adult-plant resistance to stripe rust in spring wheat cultivar Alpowa. Theor. Appl. Genet. 114:1277-1287.

Marone, D., Laidò, G., Gadaleta, A., Colasuonno, P., Ficco, D. B. M., Giancaspro, A., Giove, S., Panio, G., Russo, M. A., Vita, P. D., Cattivelli, L., Papa, R., Blanco, A., and Mastrangelo, A. M. 2012. A high-density consensus map of A and B wheat genomes. Theor. Appl. Genet. 125:1619-1638.

McIntosh, R. A., Yamazaki, Y., Dubcovsky, J., Rogers, W. J., Morris, C., Appels, R., and Xia, X. C. 2014. Catalogue of Gene Symbols for Wheat: 2013-2014 Supplement. Online publication. http://shigen.nig.ac.jp/wheat/komugi/genes/ macgene/supplement2013.pdf

Meng, L., Li, H. H., Zhang, L. Y., and Wang, J. K. 2015. QTL IciMapping: Integrated software for genetic linkage map construction and quantitative trait locus mapping in biparental populations. Crop J. 3:269-283.

Messmer, M. M., Seyfarth, R., Keller, M., Schachermayr, G., Winzeler, M., Zanetti, S., Feuillet, C., and Keller, B. 2000. Genetic analysis of durable leaf rust resistance in winter wheat. Theor. Appl. Genet. 100:419-431.

Peterson, R. F., Campbell, A. B., and Hannah, A. E. 1948. A diagrammatic scale for estimating rust intensity on leaves and stems of cereals. Can. J. Res. 26c:496-500.

Raman, H., Raman, R., Kilian, A., Detering, F., Carling, J., Coombes, N., Diffey, S., Kadlol, G., Edwards, D., McCully, M., Ruperao, P., Parkin, I., Batley, J., Luckett, D., and Wratten, N. 2014. Genome-wide delineation of natural variation for pod shatter resistance in Brassica napus. PLoS One 9:e101673.

Ren, Y., He, Z. H., Li, J., Lillemo, M., Wu, L., Bai, B., Lu, Q. X., Zhu, H. Z., Zhou, G., Du, J. Y., Lu, Q. L., and Xia, X. C. 2012a. QTL mapping of adult-plant resistance to stripe rust in a population derived from common wheat cultivars Naxos and Shanghai 3/Catbird. Theor. Appl. Genet. 125:1211-1221.

Ren, Y., Li, Z. F., He, Z. H., Wu, L., Bai, B., Lan, C. X., Wang, C. F., Zhou, G., Zhu, H. Z., and Xia, X. C. 2012b. QTL mapping of adult-plant resistances to stripe rust and leaf rust in a Chinese wheat cultivar Bainong 64. Theor. Appl. Genet. 125:1253-1262.

Roelfs, A. P., and Bushnell, W. R., eds. 1985. Diseases, Distribution, Epidemiology, and Control, Vol. 2. Academic Press, Orlando, FL.

Roelfs, A. P., Singh, R. P., and Saari, E. E. 1992. Rust Diseases of Wheat: Concepts and Methods of Disease Management. CIMMYT, México D.F., Mexico.

Rosewarne, G. M., Herrera-Foessel, S. A., Singh, R. P., Huerta-Espino, J., Lan, C. X., and He, Z. H. 2013. Quantitative trait loci of stripe rust resistance in wheat. Theor. Appl. Genet. 126:2427-2449.

Rosewarne, G. M., Singh, R. P., Huerta-Espino, J., Herrera-Foessel, S. A., Forrest, K. L., Hayden, M. J., and Rebetzke, G. J. 2012. Analysis of leaf and stripe rust severities reveals pathotype changes and multiple minor QTLs associated with resistance in an Avocet $\times$ Pastor wheat population. Theor. Appl. Genet. 124: 1283-1294.

Rosewarne, G. M., Singh, R. P., Huerta-Espino, J., William, H. M., Bouchet, S., Cloutier, S., McFadden, H., and Lagudah, E. S. 2006. Leaf tip necrosis, molecular markers and b1-proteasome subunits associated with the slow rusting resistance genes Lr46/Yr29. Theor. Appl. Genet. 112:500-508.
Schnurbusch, T., Paillard, S., Schori, A., Messmer, M., Schachermayr, G., Winzeler, M., and Keller, B. 2004. Dissection of quantitative and durable leaf rust resistance in Swiss winter wheat reveals a major resistance QTL in the Lr34 chromosome region. Theor. Appl. Genet. 108:477-484.

Scott, R. A., and Milliken, G. A. 1993. A SAS program for analysis augmented randomized complete-block design. Crop Sci. 33:865-867.

Singh, K., Ghai, M., Garg, M., Chhuneja, P., Kaur, P., Schnurbusch, T., Keller, B. and Dhaliwal, H. S. 2007. An integrated molecular linkage map of diploid wheat based on a Triticum boeoticum $\times T$. monococcum RIL population. Theor. Appl. Genet. 115:301-312.

Singh, R. P., Huerta-Espino, J., Bhavani, S., Herrera-Foessel, S. A., Singh, D. Singh, P. K., Velu, G., Mason, R. E., Jin, Y., Njau, P., and Crossa, J. 2011. Race non-specific resistance to rust diseases in CIMMYT spring wheats. Euphytica 179:175-186.

Singh, R. P., Mujeeb-Kazi, A., and Huerta-Espino, J. 1998. Lr46: A gene conferring slow-rusting resistance to leaf rust in wheat. Phytopathology 88:890-894.

Singh, R. P., Nelson, J. C., and Sorrells, M. E. 2000. Mapping Yr28 and other genes for resistance to stripe rust in wheat. Crop Sci. 40:1148-1155.

Singh, R. P., and Rajaram, S. 1992. Genetics of adult-plant resistance to leaf rust in 'Frontana' and three CIMMYT wheats. Genome 35:24-31.

Somers, D. J., Isaac, P., and Edwards, K. 2004. A high-density microsatellite consensus map for bread wheat (Triticum aestivum L.). Theor. Appl. Genet. 109:1105-1114.

Van Ooijen, J. W. 2006. Join Map 4, Software for the Calculation of Genetic Linkage Maps in Experimental Population. Plant Research International, Wageningen, The Netherlands.

Voorrips, R. E. 2002. MapChart: Software for the graphical presentation of linkage maps and QTLs. Heredity 93:77-78.

Wang, S. C., Basten, J., and Zeng, Z. B. 2012. Windows QTL Cartographer 2.5. Department of Statistics, North Carolina State University, Raleigh, NC. http://statgen.ncsu.edu/qtlcart/WQTLCart.htm

Wellings, C. R. 2011. Global status of stripe rust: A review of historical and current threats. Euphytica 179:129-141.

Wilkinson, P. A., Winfield, M. O., Barker, G. L. A., Allen, A. M., Burridge A., Coghill, J. A., Burridge, A., and Edwards, K. J. 2012. CerealsDB 2.0: An integrated resource for plant breeders and scientists. BMC Bioinf. 13: 219.

William, H. M., Singh, R. P., Huerta-Espino, J., Palacios, G., and Suenaga, K. 2006. Characterization of genetic loci conferring adult plant resistance to leaf rust and stripe rust in spring wheat. Genome 49:977-990.

William, M., Singh, R. P., Huerta-Espino, J., Ortiz Islas, S., and Hoisington, D. 2003. Molecular marker mapping of leaf rust resistance gene Lr46 and its association with stripe rust resistance gene $Y r 29$ in wheat. Phytopathology 93:153-159.

Wright, S. 1968. Genetic and Biometric Foundations, Vol. 1: Evolution and the Genetics of Populations. University of Chicago Press, Chicago.

Xu, X., Bai, G., Carver, B. F., Shaner, G. E., and Hunger, R. M. 2005. Molecular characterization of slow leaf-rusting resistance in wheat. Crop Sci. 45:758-765.

Yang, E. N., Rosewarne, G. M., Herrera-Foessel, S. A., Huerta-Espino, J., Tang, Z. X., Sun, C. F., Ren, Z. L., and Singh, R. P. 2013. QTL analysis of the spring wheat "Chapio" identifies stable stripe rust resistance despite intercontinental genotype $\times$ environment interactions. Theor. Appl. Genet. 126: 1721-1732.

Yang, H., Tao, Y., Zheng, Z., Li, C., Sweetingham, M. W., and Howieson, J. G. 2012. Application of next-generation sequencing for rapid marker development in molecular plant breeding: A case study on anthracnose disease resistance in Lupinus angustifolius L. BMC Genomics 13:318.

Zhang, L., Li, Z., Lillemo, M., Xia, X., Liu, D., Yang, W., Luo, J., and Wang, H. 2009. QTL mapping for adult-plant resistance to leaf rust in CIMMYT wheat cultivar Saar. Sci. Agric. Sin. 42:388-397.

Zhang, W., Chao, S., Manthey, F., Chicaiza, O., Brevis, J. C., Echenique, V., and Dubcovsky, J. 2008. QTL analysis of pasta quality using a composite microsatellite and SNP map of durum wheat. Theor. Appl. Genet. 117: 1361-1377. 